\title{
Real-Time Temperature Control of Thin Plates
}

\author{
Dan Necsulescu, Bilal Jarrah \\ University of Ottawa/Department of Mechanical Engineering \\ 770 King Edward ave., Ottawa, ON, Canada \\ Dan.Necsulescu@uottawa.ca; bjarr031@uottawa.ca
}

\begin{abstract}
In this paper is investigated the control of the temperature on one side of the plate from the opposite side. To solve this problem, Laplace transform is used to obtain the quadrupole model of the direct heat equation and the analytical solution for the transfer function for the inverse problem. The resulting hyperbolic functions are approximated by Taylor expansions and the real-time open loop temperature control to a desired value is formulated.

This approach is different from previous regularization methods of ill-posed problem and is suitable for real-time temperature control. Simulation results show the advantages and limitations of using inverse problem to control temperature of a plate.
\end{abstract}

Keywords: open loop control, inverse problem, plate temperature control, quadrupole model.

\section{Introduction}

The problem of temperature control of a thin metal plate is affected by the fast temperature amplitude decrease with regards to frequency and thickness. In most cases this consists in applying input temperature at one side in order to modify the temperature on the other side of the plate and in open loop control this requires to use inverse problem solution, known to lead to an ill-posed problem [1], [2]. There are many methods to address the ill-posed problem and an investigation is required to find out a suitable one for each application.

Maillet [1], Beck [2] and Necsulescu [3], in their books presented a wide variety of solutions for solving inverse heat transfer problems in case of temperature monitoring for plates. Feng et al in 2010 solved the problem of heat conduction over a finite slab to estimate temperature and heat flux on the front surface of a plate from the back surface measurement. To do this, they linked the temperature and heat flux on both sides by transfer functions and used these functions to solve the problem for heat input on the front surface of a plate, [4] and [5]. Feng and al. In 2011solvedthesame problem using a1 Dimensional (1D) modal expansion [6].Fan et al. obtained temperature distribution on one side of a flat plate by solving the inverse problem based on the temperature measurement on the other side of a plate, using the modified one-dimensional correction and the finite volume methods, [7]. Monde developed an analytical method to solve inverse heat conduction problem using Laplace transform technique [8].

In this paper the $1 \mathrm{D}$ heat conduction equation is formulated in the Laplace domain to determine the hyperbolic transfer functions relating input and output temperature of a thin plate for both direct and inverse problems. Real-time open loop control problem, which differs from the known monitoring problem, is approached using finite Taylor expansions of the hyperbolic functions. Internet-of-Things provides new interest in remote open loop control of systems.

\section{Problem Formulation}

The 1D heat conduction equation is given by:

$$
\frac{\partial^{2} \theta}{\partial \mathrm{z}^{2}}=\frac{1}{\alpha} \frac{\partial \theta}{\partial \mathrm{t}}
$$

Boundary conditions were considered the following:

$$
\begin{array}{cr}
\theta_{1}(0, \mathrm{t})=\mathrm{A} \text { sin } \omega \mathrm{t} & \theta_{2}(\mathrm{~L}, \mathrm{t})=\text { free } \\
\emptyset_{1}(0, \mathrm{t})=\text { free } & \emptyset_{2}(\mathrm{~L}, \mathrm{t})=0
\end{array}
$$


where $\theta$ is the temperature and $\emptyset$ is the heat flux.

These boundary conditions were chosen for the investigation of temperature control with sinusoidal input $\theta_{1}(0, t)$ resulting in the temperature output $\theta_{2}(L, t)$ on the opposite side of a plate of thickness L. The heat flux $\emptyset_{1}(0, t)$ results from the imposed $\theta_{1}(0, t)$, while heat flux $\emptyset_{2}(L, t)$ corresponds to isolated side of the plate.

Applying Laplace transform, this equation becomes:

$$
\frac{\mathrm{d}^{2} \theta(\mathrm{z}, \mathrm{s})}{\mathrm{dz}}=\frac{\mathrm{s}}{\alpha} \theta(\mathrm{z}, \mathrm{s})
$$

where $\alpha$ is the thermal diffusivity.

The solution of this equation is [2,3]:

$$
\theta(\mathrm{z}, \mathrm{s})=\mathrm{A}_{1} \cosh (\mathrm{Kz})+\mathrm{A}_{2} \sinh (\mathrm{Kz})
$$

where $\theta$ is the temperature.

The heat flux is given by:

$$
\emptyset(\mathrm{z}, \mathrm{s})=-\mathrm{ks} \frac{\mathrm{d} \theta}{\mathrm{ds}}
$$

where

$$
\mathrm{K}=\sqrt{\frac{\mathrm{s}}{\alpha}}
$$

These equations define the thermal quadrupole given the defined boundary conditions [1], in this case:

$$
\theta_{1}(0, s)=A \frac{\omega}{S^{2}+\omega^{2}} \theta_{2}(L, s)=\text { free }
$$

where $\mathrm{A}$ is the input temperature amplitude.

$\emptyset_{1}(0, \mathrm{~s})=$ free $\emptyset_{2}(\mathrm{~L}, \mathrm{~s})=0$, where $\mathrm{L}$ is the plate thickness.

Substituting boundary conditions for temperature equation give the following results:

$$
A_{1}=A \frac{\omega}{S^{2}+\omega^{2}} \quad, A_{2}=-A \frac{\omega}{S^{2}+\omega^{2}} \tanh (K L)
$$

For the above $A_{1}$ and $A_{2}$ the solutions become:

$$
\begin{gathered}
\theta(\mathrm{z}, \mathrm{s})=\mathrm{A} \frac{\omega}{\mathrm{S}^{2}+\omega^{2}}[\cosh (\mathrm{Kz})-\tanh (\mathrm{KL}) \sinh (\mathrm{Kz})] \\
\varnothing(\mathrm{z}, \mathrm{s})=-\mathrm{ksKA} \frac{\omega}{\mathrm{S}^{2}+\omega^{2}}[\cosh (\mathrm{Kz})-\tanh (\mathrm{KL}) \sinh (\mathrm{Kz})]
\end{gathered}
$$

where $\mathrm{k}$ is the thermal conductivity.

For the investigation of the dynamics of temperatures $\theta_{1}$ and $\theta_{2}$, the following equations are used:

$$
\begin{gathered}
\theta_{1}=\theta(0, \mathrm{~s})=\mathrm{A} \frac{\omega}{\mathrm{S}^{2}+\omega^{2}} \\
\theta_{2}=\theta(\mathrm{L}, \mathrm{s})=\mathrm{A} \frac{\omega}{\mathrm{S}^{2}+\omega^{2}}[\cosh (\mathrm{KL})-\tanh (\mathrm{KL}) \sinh (\mathrm{KL})]=\mathrm{A} \frac{\omega}{\mathrm{S}^{2}+\omega^{2}}[1 / \cosh (\mathrm{KL})]
\end{gathered}
$$


The transfer function of the direct problem linking $\theta_{1}$ and $\theta_{2}[3]$ is:

$$
\mathrm{G}_{1}=\frac{\theta_{2}}{\theta_{1}}=\left[\frac{1}{\cosh (\mathrm{KL})}\right]=\operatorname{sech}(\mathrm{KL})
$$

The transfer function for the inverse problem [1] to [3] is:

$$
\mathrm{G}_{2}=\frac{1}{\mathrm{G}_{1}}=\cosh (\mathrm{KL})
$$

The open loop control block diagrams shown in Fig. 1.

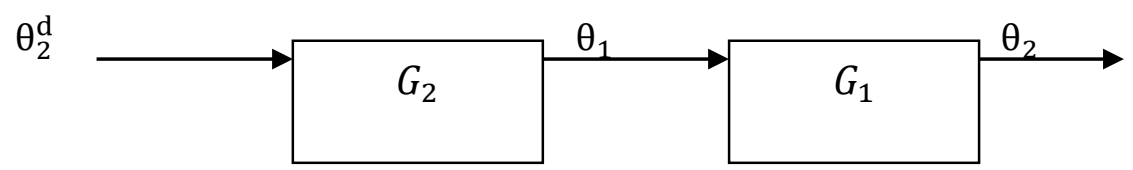

Fig. 1: Open loop block diagram.

MATLAB $^{\mathrm{TM}}$ and Simulink ${ }^{\mathrm{TM}}$ are used for simulating the above system.

In this formulation, the hyperbolic functionsG1 and $\mathrm{G} 2$ contain square root of s:

$$
\mathrm{x}=\mathrm{KL}=\sqrt{\frac{\mathrm{s}}{\alpha} \mathrm{L}}
$$

Taylor series expansion provides equations in integer powers of $\mathrm{s}$. For $\mathrm{G}_{1}=\operatorname{sech}(\mathrm{x})$ Taylor series expansion is:

$$
G_{1}=\operatorname{sech}(x)=\sum_{n=0}^{\infty}\left(\frac{E_{n}}{n !}\right) x^{n} \text { for }|x|<\frac{\pi}{2}
$$

where Euler numbers $E_{n}$ are zero for odd-indexed numbers, while even indexed numbers are:

$$
\begin{aligned}
& E_{0}=1 \\
& E_{2}=-1 \\
& E_{4}=5 \\
& E_{6}=-61 \\
& E_{8}=1385 \\
& E_{10}=-50521 \\
& E_{12}=2702765 \\
& E_{14}=-199360981 \quad \text { etc. }
\end{aligned}
$$

For $\mathrm{G}_{2}=\cosh (\mathrm{x})$, also an even function results:

$$
G_{2}=\cosh (x)=\sum_{n=0}^{\infty}\left(\frac{1}{(2 n) !}\right) x^{2 n} \quad \text { for }-\infty<x<\infty
$$

The above Taylor series expansions of $1 / \mathrm{G} 1=\cosh (\mathrm{x})$ and $\mathrm{G} 2=\cosh (\mathrm{x})$ contain only even-indexed terms, and give integer number powers polynomials in s for simulation. This polynomial approximation, easy to compute, is particularly useful in real-time control of the plate temperature.

For the Simulink simulation, Taylor expansion of the direct problem transfer function, $\mathrm{G}_{1}$, will be limited to $\mathrm{N}$ terms, and inverse problem transfer function, $\mathrm{G}_{2}$, is limited to $\mathrm{M}$ terms. For the transfer function $\mathrm{G} 1{ }^{*} \mathrm{G} 2, \mathrm{~N}$ and $\mathrm{M}$ are chosen such that $\mathrm{N} \geq \mathrm{M}$. In the simulated case, here a thin Aluminum plate, has the thickness $\mathrm{L}=0.03[\mathrm{~m}]$ and thermal diffusivity $\alpha=$ $9.715 \mathrm{e}-5\left[\mathrm{~m}^{2} / \mathrm{sec}\right]$, such that: 


$$
\mathbf{x}=\mathrm{KL}=\sqrt{\frac{\mathrm{s}}{\alpha}} \mathrm{L}=\sqrt{\frac{\mathrm{s}}{9.715 * 10-5}} 0.03
$$

For the simulations was chosen $\mathrm{M}=4$ and $\mathrm{M}=8$, i.e. $\mathrm{N}>\mathrm{M}$ :

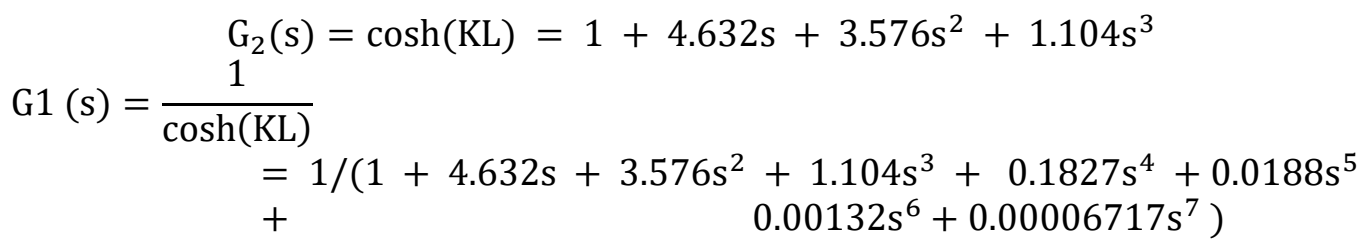

\section{Results and Discussion}

Simulations were carried out for open loop control for different values of input frequency and for the desired sinusoidal temperature amplitude of $20^{\circ}$ above the original temperature, i.e., for $20 \sin \omega t$.

Simulations were carried for the direct problem $\mathrm{G} 1$ for $\mathrm{M}=8$ while for inverse problem $\mathrm{G} 2$ for $\mathrm{M}=4$. The input was $\theta_{2}^{\mathrm{d}}=$ $20 \sin (\omega t)$.Simulation results for $\omega=0.1,1,5$, and 10 are shown in Fig. 2-6

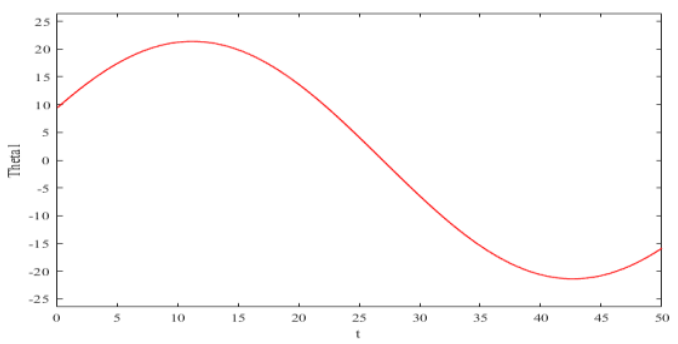

(a)

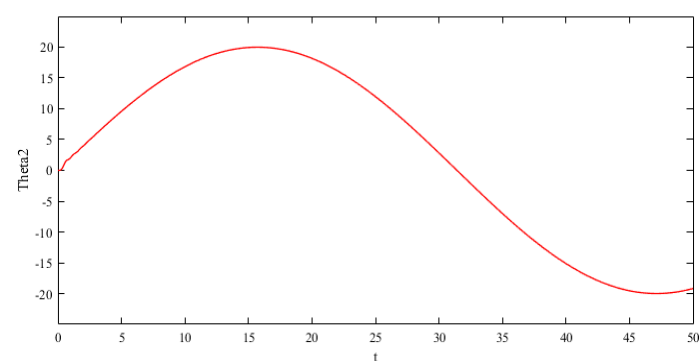

(b)

Fig. 2: The outputs (a) of inverse problem $\theta_{1}$ and of (b) open loop control $\theta_{2}$ for $\omega=0.1 \mathrm{rad} / \mathrm{sec}$.

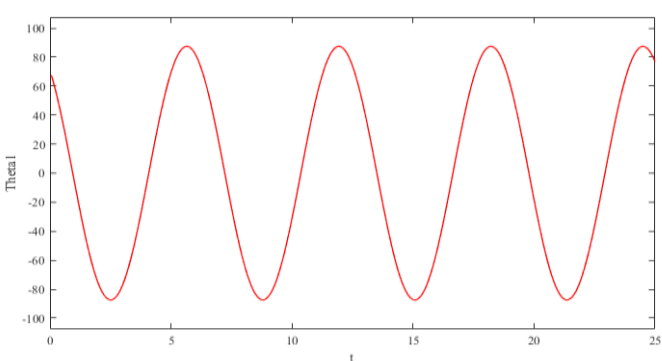

(a)

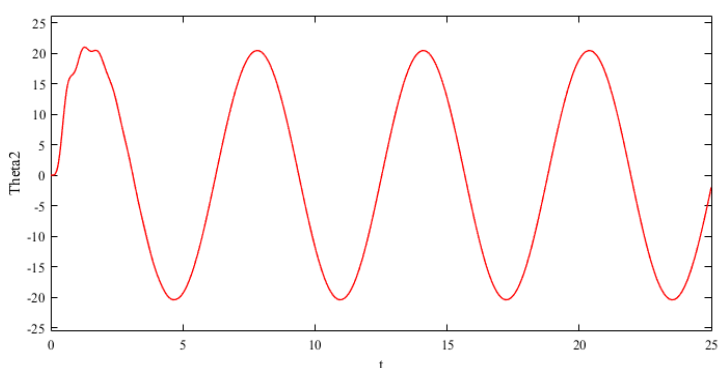

(b)

Fig. 3: The outputs (a) of inverse problem $\theta_{1}$ and (b) of open loop control $\theta_{2}$ for $\omega=1 \mathrm{rad} / \mathrm{sec}$.

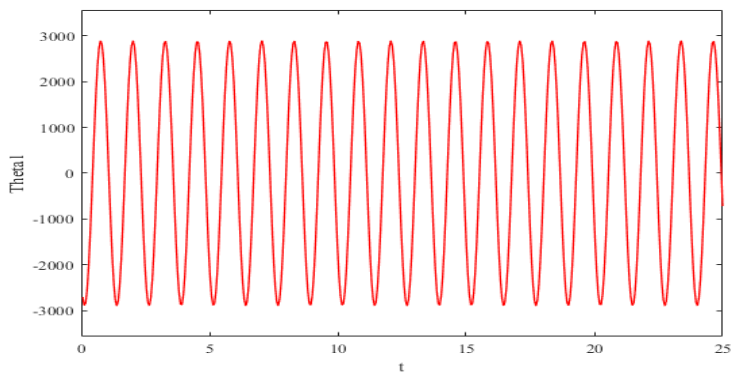

(a)

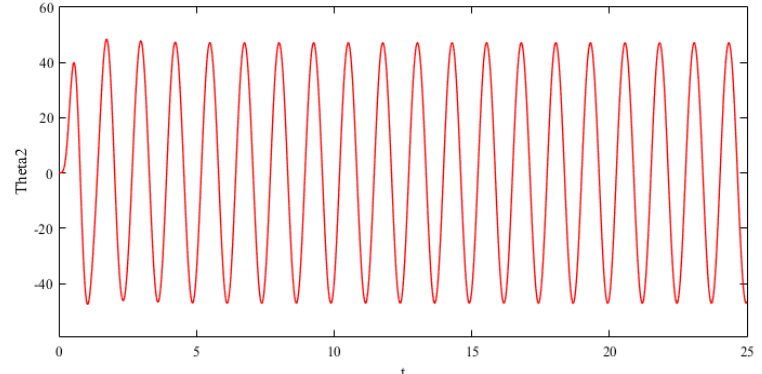

(b)

Fig. 4: The outputs (a) of inverse problem $\theta_{1}$ and (b) of open loop control $\theta_{2}$ for $\omega=5 \mathrm{rad} / \mathrm{sec}$. 


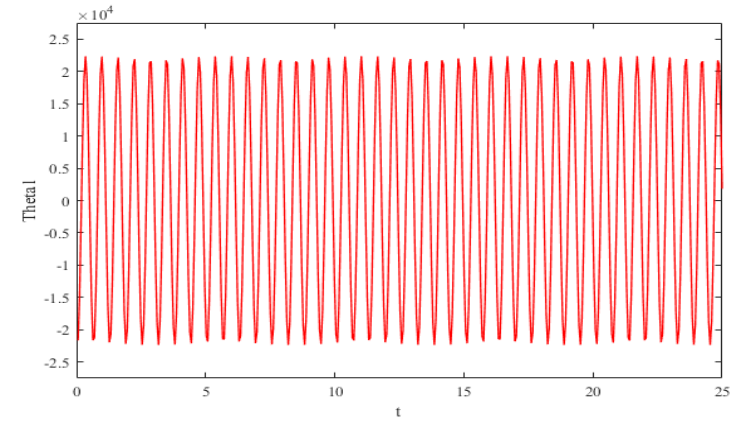

(a)

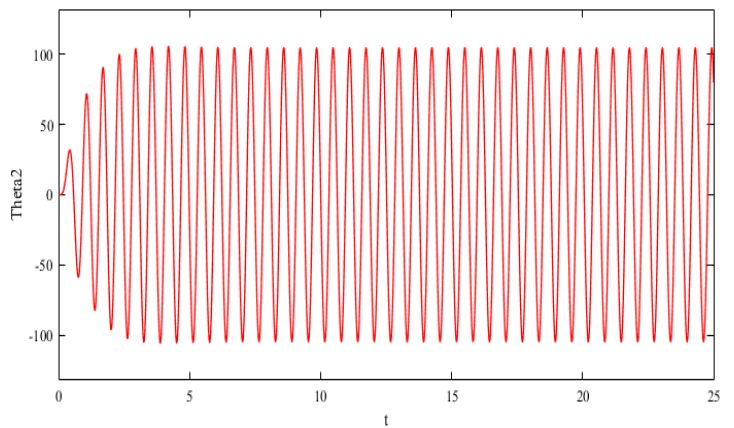

(b)

Fig. 5: The outputs (a) of inverse problem $\theta_{1}$ and (b) of open loop control $\theta_{2}$ for $\omega=10 \mathrm{rad} / \mathrm{sec}$.

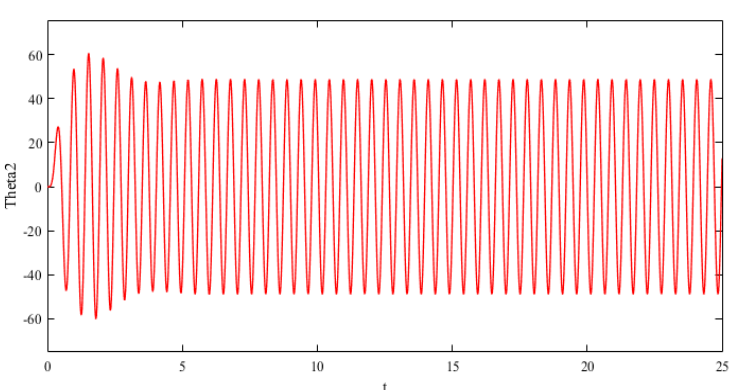

(a)

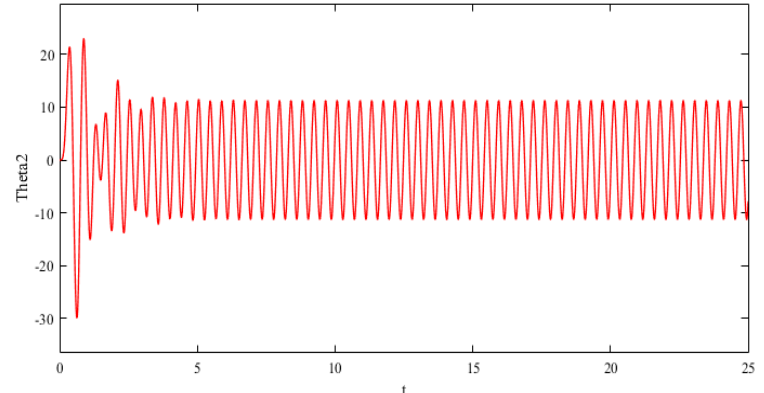

(b)

Fig. 6: The output of open loop control with $\mathrm{N}=8, \mathrm{M}=4$ for (a), $\omega=12$ and (b) $15 \mathrm{rad} / \mathrm{sec}$.

Fig. 7 shows the Bode diagram of open loop control transfer function $\mathrm{G} 1 * \mathrm{G} 2$ for $\mathrm{N}=8$ and $\mathrm{M}=4$.

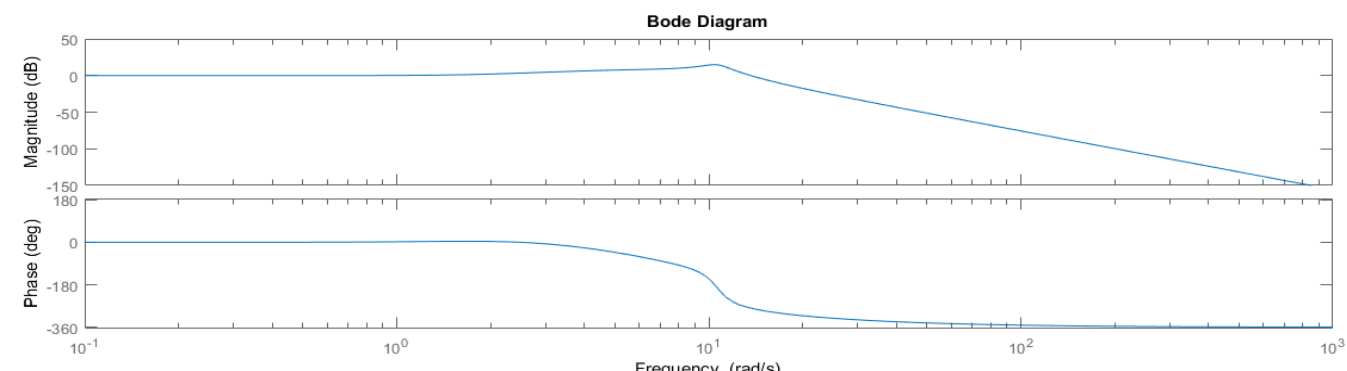

Fig. 7: Bode diagram of open loop control transfer function $\mathrm{G} 1 * \mathrm{G} 2$ for $\mathrm{N}=8$ and $\mathrm{M}=4$.

The simulation results in Fig. 2-5 represent (a) the outputs of the inverse problem $\theta_{1}$ and (b) of open loop control $\theta_{2}$ with $\mathrm{N}=8, \mathrm{M}=4$ for $\omega=0.1,1,5$ and $10 \mathrm{rad} / \mathrm{sec}$. The output temperature $\theta_{2}$ results in Fig 2 and 3 , for lower frequencies of 0.1 and $1 \mathrm{rad} / \mathrm{sec}$, compared to desired one, $\theta_{2}^{\mathrm{d}}=20 \sin \omega \mathrm{t}$, and of the command temperature $\theta_{1}$, are very close. The results in Fig 4 and 5, for output temperature $\theta_{2}$, for higher frequencies of 5 and $10 \mathrm{rad} / \mathrm{sec}$, compared to desired one, $\theta_{2}^{\mathrm{d}}=$ 20 sin $\omega t$, and of the command temperature $\theta_{1}$, are significantly different. This can be explained by the very high amplitudes of the output of the inverse problem in fig. $4 \mathrm{a}$ and $5 \mathrm{a}$, which lead eventually to an ill-posed inverse problem, particularly with regard to parameters L and $\alpha$ uncertainty. For $\omega=12$, Fig. 6a shows an amplitude lower than in Fig 5b and for $\omega=15$, shows an amplitude much lower than in Fig 5b. Bode diagram of open loop control transfer function in Fig. 7 explains this by indicating significantly lower magnitudes for $\omega>11 \mathrm{rad} / \mathrm{sec}$.In open loop control, since there is no feedback from the output, parameter uncertainty and disturbance effects cannot be reduced. Further study will focus on closed loop temperature control. 


\section{Conclusions}

The temperature on the one face of a plate can be open loop controlled in real-time to a desired value from the other face using the proposed polynomial approach. The resulting hyperbolic functions are approximated by Taylor expansions. The proposed approach is different from previous regularization methods of ill-posed problems due to using truncated polynomials and is particularly suitable for real-time open loop temperature control. Simulation results indicate the advantages and the limitations of the proposed approach.

\section{References}

[1] D. Maillet, S. Andre, J. Batsale, A. Degiovanni, and C. Moyne, "Thermal Quadrupoles," in Solving the Heat Equation through integral Transform. England: Wiley\& Sons, 2000.

[2] J. Beck, B. Blackwell, and C. Clair, Inverse Heat Conduction. John Wiley \& Sons, 1985.

[3] D. Necsulescu, "Advanced Mechatronics, Monitoring and Control of Spatially Distributed Systems," World Scientific, 2009.

[4] Z. Feng, J. Chen, Y. Zhang, and S. Montgomery-smith, "Temperature and heat flux estimation from sampled transient sensor measurements," Int. Journal of Thermal Sciences, pp. 2385-2390, 2010.

[5] Z. Feng, J. Chen, and Y. Zhang, "Real-time solution of heat conduction in a finite slab for inverse analysis," International Journal of Thermal Science, vol. 49, pp. 762-768, 2010.

[6] Z. Feng, J. Chen, Y. Zhang, and J. Griggs, "Estimation of front surface temperature and heat flux of a locally heated platefrom distributed sensor data on the back surface," International Journal of Heat and Mass Transfer, vol. 54, pp. 3431-3439, 2011.

[7] C. Fan, F. Sun, and L. Yang, "A simple method for inverse estimation of surface temperature distribution on a flat plate," Inverse Problems in Science and Engineering, vol. 17, no. 7, pp. 885-899, 2009.

[8] M. Monde, "Analytical method in inverse heat transfer problem using Laplace transform technique," International Journal of Heat and Mass Transfer, vol. 43, pp. 3965-3975, 2000. 